\title{
The Psychiatry Access Center: A New Opportunity for Innovative Residency Training
}

\author{
M. Bates Redwine M.D. \\ Medical university of South Carolina, Department of Psychiatry and Behavioral Sciences, MUSC Instutute \\ of Psychiatry, Access Center, Charleston, SC
}

Ryan Finkelbine M.D.

Medical university of South Carolina, Department of Psychiatry and Behavioral Sciences, MUSC Instutute of Psychiatry, Access Center, Charleston, SC

William Carson M.D.

Medical university of South Carolina, Department of Psychiatry and Behavioral Sciences, MUSC Instutute of Psychiatry, Access Center, Charleston, SC

Susan Hardesty M.D.

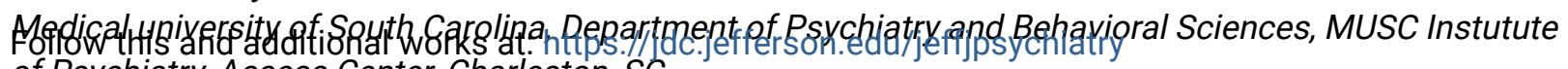
of Psychiatry, Access Center, Charleston, SC

Part of the Psychiatry Commons

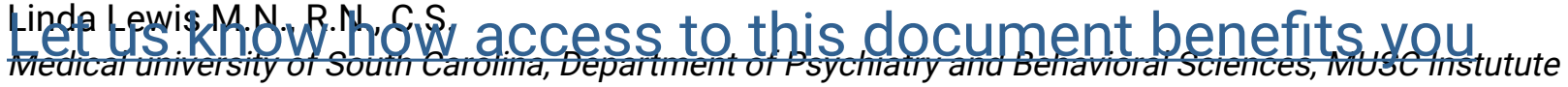
of Psychiatry, Áccess Center, Charieston, SC

\section{Recommended Citation}

Redwine, M. Bates M.D.; Finkelbine, Ryan M.D.; Carson, William M.D.; Hardesty, Susan M.D.; and Lewis, Linda M.N., R.N., C.S. (1999) "The Psychiatry Access Center: A New Opportunity for Innovative Residency Training," Jefferson Journal of Psychiatry. Vol. 14 : Iss. 2 , Article 5.

DOI: https://doi.org/10.29046/JJP.014.2.002

Available at: https://jdc.jefferson.edu/jeffjpsychiatry/vol14/iss2/5

This Article is brought to you for free and open access by the Jefferson Digital Commons. The Jefferson Digital Commons is a service of Thomas Jefferson University's Center for Teaching and Learning (CTL). The Commons is a showcase for Jefferson books and journals, peer-reviewed scholarly publications, unique historical collections from the University archives, and teaching tools. The Jefferson Digital Commons allows researchers and interested readers anywhere in the world to learn about and keep up to date with Jefferson scholarship. This article has been accepted for inclusion in Jefferson Journal of Psychiatry by an authorized administrator of the Jefferson Digital Commons. For more information, please contact: JeffersonDigitalCommons@jefferson.edu. 


\title{
The Psychiatry Access Center: A New Opportunity for Innovative Residency Training
}

\author{
M. Bates Redwine, M.D., Ryan Finkenbine, M.D., \\ William H. Carson, M.D., Susan Hardesty, M.D. \\ and Linda Lewis, M.N., R.N., C.S.
}

\begin{abstract}
A recently introduced $P G Y-1$ rotation in an innovative, managed care oriented program offers many training advantages for residents. The rotation offers first-year residents intensive interviewingskills training, experience with obtaining and documenting the psychiatric history and physical examination, and in the process, offers early exposure to a managed care environment. The Psychiatry Access Center employs non-resident clinicians to perform urgent evaluations and all admissions to the main psychiatric hospital. The service has been tested successfully without requiring resident work, and therefore allows the integration of residents into the system via a new rotation designed to focus exclusively on educational needs.
\end{abstract}

\section{INTRODUCTION}

In the era of managed care many academic environments in which residents are trained have changed dramatically. The economic forces driving change have led to greater work responsibilities for residents with few associated educational benefits. This increased service role for residents has come at a time when fewer medical students are electing to train in psychiatry, further confounding the ability to ensure a quality training experience (1). In addition, residency programs often find it difficult to provide a contemporary experience while still meeting the requirements set forth by the Accreditation Council for Graduate Medical Education (ACGME) guidelines $(2,3)$. This paper discusses our university-based, academic residency training center's approach to this problem.

\section{THE PROBLEM}

As managed care has infiltrated the academic setting, a number of changes in the clinical environment have resulted. Concern with fiscal responsibility has contrib-

Address requests for reprints to: Ryan Finkenbine, M.D., MUSC Institute of Psychiatry, Access Center, 171 Ashley Ave., Charleston, SC 29425.

Medical University of South Carolina, Department of Psychiatry and Behavioral Sciences, Charleston, South Carolina 
uted to the emphasis on crisis stabilization and led to briefer hospital stays. Day-hospital programs have become prominent. The treatment setting has shifted to provide greater outpatient services. Many of these changes, albeit economically rational, create more work. To meet greater work demands, some academic centers have depended on residents to provide service, but have not addressed the need for educational changes in parallel. That fewer students are entering the field of psychiatry adds to the problem (4). A significant concern of many training centers, therefore, has been the need to maintain a quality educational experience for psychiatric residents.

This challenge has been made even more difficult as residency programs endeavor to make patient services more clinically responsive and efficient, yet must continue to adhere to the training guidelines prescribed by the ACGME. The guidelines, in the opinion of many, have not held pace with the changes occurring in clinical care $(1,2,3)$. The problem many programs fear is that an increase in traditional residency-dependent service requirements, combined with a decrease in the number of residents and the restrictions of the ACGME guidelines, will result in an overall decline in the educational experience each resident receives.

\section{OUR SOLUTION}

At the Medical University of South Carolina's Institute of Psychiatry, the problem was first discussed by the Residency Education Committee (REG), a committee of faculty and residents that safeguards the quality of resident education and training. An important result of these discussions was the REC mandate that the delivery of the hospital's clinical service must operate independently from resident service. The goal was to continue resident involvement in patient care while ensuring that neither the hospital's service requirements nor the number of residents in the program would act as the rate-limiting factor in patient management. In other words, the REC recognized the importance of direct patient contact in residency training, but did not want the hospital's burgeoning clinical practice to overwhelm and impair the learning experience. As the influx of managed care continues, it is essential that university hospitals remain efficient regardless of the number of residents; therefore, they can no longer rely exclusively on the resident workforce.

A separate but affiliated Process Improvement Committee (PIC), which had been given the task to help ensure the continued economic soundness of clinical practice, set as one of its goals the administration of the REC's executive mandate. PIC members chose as its first task the improvement of the admissions process. Previously, a traditional responsibility of residents on inpatient wards was to admit all patients entering the hospital. This role once provided residents with significant experience in patient evaluation, diagnosis, and initiation of treatment. However, as managed care principles were applied to our setting, the number of admissions soared while the length of inpatient hospitalization shortened. Consequently, the educational benefit of admitting patients beyond a certain number leveled out. 


\section{THE MODEL}

The PIC's solution was to create an entirely new service of the department, the Psychiatry Access Center (PAC), which functions to ease patient and referrer access to all clinical and research services activities of department. The PAC also provides triage services for inpatient and partial hospitalization programs and is the central appointing entity for all outpatient services. In addition, the PAC provides urgent assessments and short-term patient management services. Patients admitted to the hospital are assessed with the completion of a formal psychiatric history and physical and all the attendant documentation prior to arrival on the inpatient ward.

All of these services are provided independent of resident help. The PAC allows patients to be seen efficiently and in large numbers without the services of residents. The PAC meets the demands of managed care, yet guarantees that residents will not be overwhelmed by service requirements.

\section{TRAINING BENEFITS}

The creation of the PAC meets the REC's mandate for making clinical services independent from residency training with regard to the admissions process. The are several training benefits of this system.

The implementation of the PAC has allowed residents to use time previously spent on admission assessments to explore a greater variety of treatment settings. Other writers have discussed the effects of managed care on training environments with regard to the constraints of brief stays and crisis-stabilization, now the focus of the current inpatient wards. Several solutions have been proposed to address this problem such as brief inpatient psychotherapy training or affiliation with partial hospitalization programs, but too often residents lack the time or flexibility to participate in a meaningful way (2). The PAC has allowed more time and flexible schedules for residents to participate in such training activities since they are no longer required to remain on the unit awaiting possible admissions. In our experience this time and flexibility has allowed better participation in traditional inpatient treatment modalities such as medication education groups and supervised brief psychotherapy, but has also enabled opportunities for new training experiences such as involvement in affiliated day hospital programs and off-campus forensics work. Additionally, residents now attend newly created longitudinal clinics in both psychiatry and primary care. As the clinical environment changes, due to response to either economic forces or to modifications in educational requirements, the greater time and flexibility in residents' schedules allowed by the PAC will ease future adaptations.

\section{THE CREATION OF A NEW TRAINING SERVICE}

The PAC created a potential training problem: residents would need additional experience with the history and physical assessment of new patients since this was 
now provided by the PAC staff. The PIC and REC recommended the creation of a new training service to address this problem. Specifically, PGY-1 residents would rotate through the PAC "to learn to complete, write, and present formal psychiatric histories and physicals, and to provide experience in the assessment, diagnosis, and disposition of a heterogenous psychiatric population across the life span and continuum of services." The PGY-1 PAC rotation was developed with these goals in mind and was introduced one year after the opening of the PAC.

We have identified several benefits since the inception of the PAC rotation. The most significant is that the PAC had been functioning for a year prior to the introduction of residents. Therefore patient services have never been dependent on resident work. Since there are no specific tasks required of residents for the PAC to function, the rotation has been designed to focus exclusively on educational needs. Residents are integrated into the PAC so that it runs smoothly while they learn new tasks and skills, yet they are not required for the service to operate efficiently.

An inherent advantage of the PAC's freedom from resident work requirements is the lack of strict guidelines (for example, every third patient) for when residents must see patients. Patients are assigned by an attending based on resident availability and need for exposure to different patient populations. A menu of core patient types has been developed to ensure a broad educational experience such that assignments are modified throughout the rotation. Residents maintain a personal log of patients that includes age, gender, race, and diagnoses. The log helps to guarantee that residents will see a wide array of patients and also serves as record to meet ACGME requirements for written documentation. The assignment process allows and encourages residents to request patients of a specific population in which they have an interest. They must meet the core requirements, but have the latitude to begin to investigate areas of professional interest. Several residents have remarked favorably that the assignment system allowed them to begin to orient towards areas of potential future study.

Another benefit of the PAC has been that residents can be paced through the one or two month rotation based on their individual educational needs. An arbitrary number of per day admissions is no longer the norm, so the frequency of patient assignments are made by the attending according to the abilities and experience of the resident. Residents are encouraged to gradually develop their assessment and presentation skills. They are pushed to remain busy, but are not overwhelmed with tedious work requirements. Assignments usually increase during the course of the rotation. A typical resident might be assigned one or two patients on the first day, but later is able to efficiently see five or more. All residents are required to see a minimum number of core patients to successfully pass the rotation, however. Residents rarely must stay beyond usual business hours to complete a particularly complex patient - and on those occasions the attending and rest of the PAC team stay also.

Time for supervision has been built into the rotation. The resident receives supervision on every patient by the attending. In addition, other PAC staff offer supervision when available, especially if they have special training or expertise in an 
applicable area. For example, one of our advanced nurse practitioners has taught graduate courses in performing a physical exam and another has special training in cognitive behavioral therapies. Since residents are gradually integrated into the independently functioning service, they reduce the total amount of work done by the entire PAC staff. This work reduction creates the time available for supervision by the attending and staff. PAC staff are required to attend mandatory training sessions, many of them taught by MUSC attendings, to maintain their skills. Sessions which focus on the supervision of trainees are regularly scheduled.

Since the PAC also provides services for patients who require urgent evaluation, but not necessarily admission, residents have gained valuable experience with non-emergent outpatient evaluations in a closely supervised setting. This training fills the void between the traditional scheduled outpatient and the assessment of patients in the emergency department. In addition, it comes early in the residency curriculum and helps to introduce trainees to the dispositional continuum.

Another advantage to the development of the new PAC rotation has been to allow residents to participate in a corollary course introduced at about the same time-a two year primary care clinic. The clinic, developed independently of the PAC, provides residents with an important long term primary care experience $(5,6)$. Each resident carries a defined patient load on a weekly basis. The PAC has allowed residents to continue this progressive training curriculum.

In order to give residents a meaningful experience with the economic practice of medicine, they are expected to complete the appropriate documentation and billing for their patients. Optional educational opportunities for residents include precertification of managed care patients and performing telephone screening of patients. Since the PAC is staffed with clinicians well versed in the criteria and processes associated with managed care financing, residents gain business acumen and should be better prepared to function as practitioners (7). Despite a call for managed care oriented education, these business skills have been under-taught in residency training (8). These activities are, of course, supervised by the attending.

Another aspect of the PAC that prepares residents for the managed care environment is its multidisciplinary staff. Residents do not often get the opportunity to work with nurse practitioners, social workers, psychologists and other nonphysician mental health clinicians in an integrated setting similar to the managed care environment. The multi disciplinary team is an essential factor in the efficiency of the PAC; residents on the rotation learn valuable information about a practice which is financially appealing to managed care providers and become comfortable working with practitioners who have varied training backgrounds $(9,10)$.

Others benefits enjoyed by residents in the PAC include: learning to administer decanoate injections, an introduction to group psychotherapy and time-limited individual psychotherapy, and, presenting a brief seminar about a topic of their choice to the PAC staff.

A final, unique, training opportunity provided by the PAC has been the introduction of a PGY-IV Chief of Service. This elective position of six to twelve months duration offers many important supervisory experiences for the resident nearing 
graduation. It can be modified to allow a role similar to PGY-I's, or remain supervisory in nature, or prepare the resident for impending private practice. Both Chiefs of Service who have taken the elective have blended these options and have also added a strong academic component.

Residents receive continuous feedback from the attending and Chief of Service throughout the rotation as well as a formal evaluation as per REC guidelines. Likewise, at the end of the rotation residents are asked to evaluate their experiences both in writing and in an exit interview. The response thus far has been positive.

Several concerns have been raised since the PAC's inception. The issue raised most often has been that of a reduction of continuity of care. While residents still follow patients on their inpatient services, and later, as long-term outpatients, there lacks the true continuum of admitting then managing their own patients. A proposal to address this problem has been introduced to the REC and involves forwarding a specific number of admissions to residents on traditional inpatient services. Another issue has been whether residents perform the same volume of assessments as in the past, before the PAC opened. Since much of the work is done by non-residents this concern is valid. It is too early to establish whether a relationship exists between quality and quantity of admissions, but the use of assessment tools to clarify the educational ramifications of this issue will be prudent. A third concern has been an underestimation of the time required of the attendings in the PAC. Whereas the introduction of residents has clearly reduced the overall time required of nonphysician staff and have allowed them to provide some supervision, the attendings have found that their supervisory role is not completely offset by resident work, especially early in the rotation. This concern has yet to be addressed.

\section{SUMMARY}

The creation of a system that uses non-resident practitioners to evaluate and admit patients to the hospital has allowed a reorganization of the manner that psychiatric services are provided. The PAC gives residents a greater variety of clinical experiences without overburdening them or slowing the admissions process. Additionally, residents are exposed to an environment similar to a managed care setting. This enables them to experience effective patient triage, third-party billing procedures, telephone screening, and elements of brief and group psychotherapy. This valuable training occurs in a setting that emphasizes interaction with non-physician mental health clinicians. In effect, since clinical services are no longer dependent on resident work, they can successfully integrate into the process in a way that emphasizes education as the primary goal.

The creation of the PAC and its associated resident rotations have not solved all of the problems that have arisen in the interface between managed care and psychiatric training, but they have presented a unique opportunity to address the problem of decreasing resident manpower in the face of increasing admissions. Many critical problems remain, including the tendency for managed care companies to exclude residents from providing psychiatric services for their patients. There is no 
question that certain essential training experiences such as long-term psychotherapy cannot be found in a managed care environment; residents will continue to need exposure to those skills in preparation for work in the "real world."

\section{REFERENCES}

1. Kay J: New challenges to the faculty in the education of psychiatrists. Bull Menninger Clin 1996;60(3):285-295.

2. Houghtalen RP, Guttmacher LB: Facilitating effective residency education on short-term inpatient units. Psychiatr Q 1996;67(2):111-124.

3. Summergrad P, Herman JB, Weilburg JB, et al: Wagons Ho: Forward on the Managed Care Trail. Gen Hosp Psychiatry 1995;17:251-259.

4. Halleck S: A different kind of education for psychiatric residents. Psychiatr Q 1996;67(2): 95-110.

5. Hales RE: Primary care in psychiatry residency training. Gen Hosp Psychiatry 1980;2:148155.

6. Lazerson AM: The psychiatrist as teacher in primary care residency training: the first year. Intl J Psychiatry in Medicine 1976-77;7:165-178.

7. Herman JB: Managed care and residency training in psychiatry. Harvard Rev Psychiatry 1995;2:290-292.

8. Beigel A, Santiago JM: Redefining the General Psychiatrist: Values, Reforms, and Issues for Psychiatric Residency Education. Psychiatr Serv 1995;46(8):769-774.

9. Blackwell B, Schmidt GL: The Educational Implications of Managed Mental Health Care. Hosp Community Psychiatry 1992;43(10):962-964.

10. Accreditation Council for Graduate Medical Education: Special requirements for residency training in psychiatry. In: Graduate Medical Education Directory 1995-1996 [monograph online]. Chicago: American Medical Association Medical Education Group; 1995 [cited 1997 Jul 23]. [13 screens]. Available from: URL:

http://www.acgme.org/acgme/progreq/psych.html. 